\title{
Joint Control
}

\section{Gemeinsame Verantwortlichkeit im Sinne der Datenschutz-Grundverordnung}

Bereits am 24. Mai 2016 trat die EU-Datenschutz-Grundverordnung in Kraft, die gemäß Art. 99 Abs. 2 S. 1 DS-GVO seit dem 25. Mai 2018 unmittelbar für alle gilt, die personenbezogene Daten im Sinne der Datenschutz-Grundverordnung verarbeiten (vgl. Art. 3 Abs. 1 DS-GVO). Mit der Datenschutz-Grundverordnung wurde zumindest für den deutschen Datenschutz das Rechtsinstitut einer sogenannten „gemeinsamen Verantwortlichkeit" neu eingeführt, welches in Art. 26 DS-GVO seine nähere Ausgestaltung gefunden hat. ${ }^{1}$ Für die zum Teil sehr komplexe Strukturen einer gemeinsamen Zusammenarbeit bei der Verarbeitung personenbezogener Daten definiert Art. 26 DS-GVO die Voraussetzungen, Regeln und zu treffenden Vorkehrungen. ${ }^{2}$

\section{Verantwortlicher}

Wer allerdings überhaupt Verantwortlicher im Sinne der Datenschutz-Grundverordnung ist, wird zunächst einmal durch Art. 4 Nr. 7 DS-GVO definiert. Danach wird als Verantwortlicher sowohl eine natürliche als auch eine juristische Person, Behörde, Einrichtung oder andere Stelle bezeichnet, die allein oder gemeinsam mit anderen über die Zwecke und Mittel der Verarbeitung von personenbezogenen Daten entscheidet. Damit erkennt die Datenschutz-Grundverordnung an, dass die Entscheidungsbefugnis über die Verarbeitung personenbezogener Daten durchaus von verschiedenen Verantwortlichen gemeinsam getragen werden kann, mit der Folge, dass auch die rechtliche Verantwortung für diese Datenverarbeitung verschiedenen Personen bzw. Stellen obliegt. ${ }^{3}$ Dies dient nicht zuletzt der klaren Zurechnung von Verantwortlichkeiten und der Rechtssicherheit aller Beteiligten im Rahmen zumeist sehr komplexer Strukturen einer Zusammenarbeit. ${ }^{4}$

\section{Joint Controller}

Wer eigentlich ein "gemeinsamer Verantwortlicher" und damit ein sogenannter „Joint Controller" ist, lässt sich demgegenüber dem Art. 26 Abs. 1 Satz 1 DS-GVO entnehmen, wonach zwei oder mehrere Verantwortliche gemeinsam die Zwecke der Ver-

1 Dazu, dass es in der EG-Datenschutzrichtlinie (RL 95/46/EG) zwar schon angelegt, allerdings nicht ausreichend ausgestaltet war, vgl. DSK-Kurzpapier Nr. 16, zu finden unter https://www.lda.bayern.de/media/dsk_kpnr_16_gemein sam_ verantwortliche.pdf (letzter Abruf 29.1.2018); zur Entstehungsgeschichte außerdem Kartheuser/Nabulsi, MMR 2018, S. 717.

2 Vgl. Hartung, in: Kühling/Buchner (Hrsg.), DS-GVO Kommentar, München 2017, Art. 26 Rn. 1.

3 Hierzu näher bei Hartung, in: Kühling/Buchner (Hrsg.), DS-GVO Kommentar, München 2017, Art. 4 Nr. 7 Rn. 12.

4 Ebenso Lang, in: Taeger/Gabel (Hrsg.), DSGVO BDSG Kommentar, 3. Aufl. 2019, Art. 26 Rn. 2; zum komplexen Zusammenspiel verschiedener Verantwortlicher vgl. aber vor allem bei Öztürk, DuD 2019, Heft 3, S. 143, in diesem Heft; oder bereits in https://www.datenschutz-notizen.de/joint-control-bei-der-arbeitnehmerueberlassung-eine-datenschutzkonforme-loesung-4721053/ (letzter Abruf 1.2.2019). arbeitung und die Mittel zu dieser Verarbeitung festlegen müssen. Es muss sich demnach um eine gemeinsame Entscheidung über die Zwecke und Mittel handeln, weshalb jeder der Beteiligten einen bestimmenden tatsächlichen Einfluss (wenn auch nicht zwingend gleichrangig) auf die Datenverarbeitung haben muss. ${ }^{5}$

$\mathrm{Zu}$ berücksichtigen ist insoweit aber, dass die gemeinsame Verantwortlichkeit weder Grundlage einer Entscheidung der beteiligten Personen oder Stellen ist, noch einfach durch eine entsprechende Vereinbarung begründet wird. ${ }^{6}$ Vielmehr richtet sich die Feststellung einer gemeinsamen Verantwortlichkeit danach, inwieweit die genannten Kriterien objektiv erfüllt sind. ${ }^{7}$ Eine entsprechende Entscheidungsgewalt bzgl. der Verarbeitung muss demzufolge anhand rechtlicher und tatsächlicher Umstände wirklich ermittelt werden können. ${ }^{8}$

Durch die gemeinsame Verantwortlichkeit tritt jedoch keine Privilegierungswirkung ein, d. h. untereinander sind die gemeinsamen Verantwortlichen weiterhin Empfänger der Daten im Sinne von Art. 4 Nr. 9 DS-GVO und deren Übermittlung bedarf einer eigenen Rechtsgrundlage (bspw. in Form einer Einwilligung). ${ }^{9}$

Vereinbarung

Wichtig ist, dass sofern das Vorliegen einer gemeinsamen Verantwortlichkeit festgestellt werden kann, gemäß Art. 26 Abs. 1 Satz 2 DS-GVO die Verantwortlichen eine Vereinbarung zu schließen haben, in denen die untereinander vorgenommene Verteilung der von den Verantwortlichen zu erfüllenden Pflichten für die betroffenen Personen transparent geregelt ist. Gegebenenfalls kann überdies eine Datenschutz-Folgenabschätzung für die Datenverarbeitung notwendig werden..$^{10}$ Die Nichteinhaltung der mit einer gemeinsamen Verantwortlichkeit einhergehenden Verpflichtungen kann ansonsten nach Art. 83 Abs. 4 lit. a DS-GVO zu einer Ahndung durch die Aufsichtsbehörden führen. ${ }^{11}$

5 Vgl. DSK-Kurzpapier Nr. 16, zu finden unter https://www.Ida.bayern.de/media/dsk_kpnr_16_gemein sam_verantwortliche.pdf (letzter Abruf 29.1.2018).

6 Hierzu auch bei Lang, in: Taeger/Gabel (Hrsg.), DSGVO BDSG Kommentar, 3. Aufl. 2019, Art. 26 Rn. 13 m. w. Nw.

7 Hierzu auch bei Lang, in: Taeger/Gabel (Hrsg.), DSGVO BDSG Kommentar, 3. Aufl. 2019, Art. 26 Rn. 13 m. w. Nw.

8 Lang, in: Taeger/Gabel (Hrsg.), DSGVO BDSG Kommentar, 3. Aufl. 2019, Art. 26 Rn. 17 ff.; zur Abgrenzung der Auftragsverarbeitung, bei der die Weisungsbefunis bzgl. des Verantwortlichen festzustellen ist, vgl. Schwerpunktthema in diesem Heft, hierzu u. a. Seiter, DuD 2019, Heft 3; S. 127; Bleckmann, DuD 2019, Heft 3, S. 137; Conrad, DuD 2019, Heft 3, S. 134, alle in diesem Heft.

9 Hierzu DSK-Kurzpapier Nr. 16, zu finden unter https://www.lda.bayern.de/ media/dsk_kpnr_16_gemein sam_verantwortliche.pdf (letzter Abruf 29.1.2018).

10 Vgl. DSK-Kurzpapier Nr. 16, zu finden unter https://www.Ida.bayern.de/ media/dsk_kpnr_16_gemein sam_verantwortliche.pdf (letzter Abruf 29.1.2018).

11 Zu möglichen Regelungsinhalten bei Arbeitnehmerüberlassung siehe Öztürk, https://www.datenschutz-notizen.de/joint-control-bei-der-arbeitnehmerueberlassung-eine-datenschutzkonforme-loesung-4721053/ (letzter Abruf 1.2.2019). 